\title{
An Evidence-based Approach to Using Intravitreal Steroids in the Management of Diabetic Macular Oedema
}

\author{
Mehmet Ozturk, Martin L Harris and Hemal Mehta \\ Royal Free London NHS Foundation Trust, London, UK
}

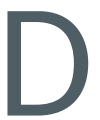
iabetic macular oedema (DMO) is the leading cause of acquired visual loss in the working age population. The landscape for DMO treatment has changed significantly over the past decade. Macular laser has been reported to reduce the risk of moderate visual loss in seminal clinical trials from the 1980s, but relatively few patients achieved visual gain. With the advent of intravitreal pharmacotherapy it is now possible to achieve visual gains in the majority of patients. Intravitreal anti-vascular endothelial growth factor (VEGF) agents are now a first-line treatment option in centre-involving DMO. This review assesses clinical trial and more recent real-world evidence to guide clinicians as to when intravitreal steroids should be considered in the management of DMO. In particular, intravitreal steroids can be considered in pseudophakic patients, or those due to undergo cataract surgery, in patients unable to attend for regular intravitreal procedures, and non-responders to intravitreal anti-VEGF therapy. Assessing clinical trial data, the dexamethasone implant appears to have a more predictable intraocular rise profile than triamcinolone or fluocinolone with a lower requirement for incisional glaucoma surgery. There is a need for consensus regarding real-world outcome measures for intravitreal steroids in the management of DMO to allow easier comparison across studies.

\section{Keywords}

Steroid, diabetic macular oedema, real-world evidence, clinical trials, triamcinolone,

fluocinolone, dexamethasone

Disclosure: Hemal Mehta is an invited member of the Allergan International Retinal Panel. He was also an investigator on the BEVORDEX randomised clinical trial. Mehmet Ozturk and Martin Harris have nothing to disclose in relation to this article.

Compliance with Ethics: This study involves a review of the literature and did not involve any studies with human or animal subjects performed by any of the authors.

Authorship: All named authors meet the International Committee of Medical Journal Editors (ICMJE) criteria for authorship of this manuscript, take responsibility for the integrity of the work as a whole, and have given final approval to the version to be published.

open Access: This article is published under the Creative Commons Attribution Noncommercial License which permits any non-commercial use, distribution, adaptation and reproduction provided the original author(s) and source are given appropriate credit.

Received: 11 May 2017

Accepted: 9 June 2017

Citation: European Ophthalmic Review, 2017;11(1):44-54

Corresponding Author: Hemal Mehta, Royal Free London NHS Foundation Trust, Pond Street, Hampstead, London, NW3 2QG, UK. E: HM@cantab.net

Support: The publication of this article was supported by Allergan. The views and opinions expressed are those of the authors and do not necessarily reflect those of Allergan.
Diabetic macular oedema (DMO) is the leading cause of acquired visual loss in the working age population. ${ }^{1}$ There are estimated to be 21 million people with DMO globally, ${ }^{2}$ with numbers expected to rise as diabetes becomes more common and life expectancy improves. ${ }^{3}$

The landscape for DMO treatment has changed significantly over the past decade. Macular laser photocoagulation was reported to be effective in reducing the rate of moderate visual loss in seminal large scale clinical trials such as the Early Treatment Diabetic retinopathy Study (ETDRS). ${ }^{4}$ Management of centre-involving DMO was subsequently revolutionised with the introduction of intravitreal therapies such as corticosteroids and anti-vascular endothelial growth factor (antiVEGF) inhibitors, where it was not only possible to prevent visual loss but also to potentially improve vision. ${ }^{5,6}$ There is clinical trial evidence to support intravitreal steroid therapy (triamcinolone, fluocinolone or dexamethasone) as a treatment option for the management of $\mathrm{DMO}$; however, local side-effects of accelerated cataract formation and raised intraocular pressure (IOP) need to be managed.? Intravitreal anti-VEGF therapy has become the mainstay of treatment for DMO, but the potential for increased rates of thromboembolic events in high risk populations, ${ }^{8}$ insufficient treatment response in some patients ${ }^{9}$ and the treatment burden ${ }^{10}$ from frequent injections remain potential disadvantages.

This review aims to supplement evidence from clinical trials of intravitreal steroid therapy with more recent real-world data to provide guidance on their role in the management of DMO.

\section{Triamcinolone}

\section{Clinical trials}

The Triamcinolone for Diabetic Macular Oedema (TDMO) study by Gillies et al. was a 2-year, randomised, double-masked, placebo-controlled trial of intravitreal triamcinolone in 69 eyes (43 patients) with DMO unresponsive to macular laser therapy (MLT). ${ }^{11}$ The patients were split into the intravitreal triamcinolone acetonide (IVTA) group ( $n=34$ eyes), receiving 4 mg of IVTA (mean 2.6 injections over 2 years), and the control group ( $n=35$ eyes), who received subconjunctival saline placebo injections (mean 1.8 injections over 2 years). The primary outcome measure was increase in best corrected visual acuity (BCVA) by $\geq 5$ logMAR letters. BCVA increase was observed in $56 \%$ in the IVTA group and $26 \%$ in the control group $(p=0.006$ ). Furthermore, at 2 years the IVTA group had a mean 3.1 letter gain in BCVA whereas the control group had a 2.9 letter loss. With regard to central macular thickness (CMT), the IVTA group had a decrease of $125 \mu \mathrm{m}$ compared to $75 \mu \mathrm{m}$ in the control group. In terms of adverse events, cataract surgery was required in $54 \%$ of patients in the IVTA group compared with $0 \%$ in the control group. IOP rise of $>5 \mathrm{mmHg}$ was observed in 
$68 \%$ of eyes in the IVTA group compared with $10 \%$ in the control group. In addition, $5.9 \%$ of eyes in the IVTA group required trabeculectomy surgery. Following the 2-year endpoint, the TDMO study became open label and patients randomised to the control group (placebo) could commence treatment with triamcinolone. ${ }^{12}$ At 5 years, BCVA increase $\geq 5$ letters was observed in $42 \%$ of those initially in the IVTA group and $32 \%$ in the original placebo group, but this difference was not statistically significant $(p=0.4)$. Similarly, no significant difference between the groups in mean CMT reduction was observed at 5 years. Furthermore, the proportion of patients in the initial IVTA group requiring cataract and trabeculectomy surgery was $71 \%$ and $9 \%$ respectively by 5 years.

The Diabetic Retinopathy Clinical Research (DRCR) Network Protocol $\mathrm{B}$ is an American multicentre, three-arm, randomised, controlled trial assessing the safety and efficacy of triamcinolone in DMO. ${ }^{13}$ Patients were divided into three groups; group 1 received $1 \mathrm{mg}$ of intravitreal triamcinolone (IVTA1, $n=256$ eyes), group 2 received $4 \mathrm{mg}$ of intravitreal triamcinolone (IVTA4, $n=254$ eyes) and group 3 were treated with focal/ grid laser photocoagulation ( $L, n=330$ eyes). The follow-up duration was 2 years. Retreatment if indicated was performed at least 3.5 months after the previous treatment for persistent DMO. Baseline visual acuity (VA) was 59 ETDRS letters (standard deviation [SD] 11), at the 2-year endpoint this changed by -2 (SD 18; $p=0.02),-3$ (SD 22; $p=0.002$ ) and +1 (SD 17) in the IVTA1, IVTA4 and $L$ groups, respectively. The proportion of patients with a BCVA gain $\geq 10$ letters in the IVTA1 group, IVTA4 group and laser group at 2 years was $25 \%, 28 \%$ and $31 \%$, respectively. There was no significant difference in results with subgroup analysis of pseudophakic patients or in those with early cataract. Changes in CMT from baseline at

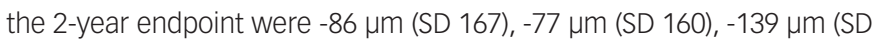
148) in the IVTA1, IVTA4 and L groups, respectively. In terms of number of treatments IVTA1, ITVA4 and $L$ groups required an average of 3.5, 3.1 and 2.9 treatments, respectively. At the 3-year endpoint, $83 \%$ of the IVTA4 group needed cataract surgery compared with $46 \%$ in the IVTA1 group and $31 \%$ the laser group. The proportion of patients who had an IOP rise of $>10 \mathrm{mmHg}$ from baseline was $18 \%$, 33\% and $4 \%$ in IVTA1, IVTA4 and laser groups, respectively over a 3-year period. Five percent of the IVTA4 group required glaucoma surgery compared with none in IVTA1 and the laser group. In a subgroup analysis of patients with severe visual impairment defined as BCVA $6 / 60-6 / 96,77 \%$ of the IVTA4 group had an improvement of $\geq 10$ letters compared to $42 \%$ receiving macular laser. The strengths of this study are the duration of follow-up, its prospective nature and the relatively large number patients enrolled. However, the 2-year completion rate excluding deaths was $88 \%$ and only $36 \%$ at 3 years.

DRCRnet Protocol B13 had different baseline characteristics compared with the TDMO trial," having excluded eyes which investigators considered unlikely to benefit from macular laser treatment and therefore including eyes with on average milder disease. This may account for the contradictory results of the TDMO and DRCRnet Protocol B trials. A small sub-group analysis of patients in DRCRnet Protocol B with severe visual impairment at baseline (BCVA 6/60-6/96) demonstrated that $77 \%$ of the IVTA4 group versus $42 \%$ in the laser group experienced $\geq 10$ letter improvement. ${ }^{13}$

The DRCRnet Protocol I study compared three groups; 4 mg of intravitreal triamcinolone (Trivaris; Allergan, New Jersey, US) and combined laser, laser alone and ranibizumab with prompt or deferred laser in patients with centre-involving DMO. ${ }^{14}$ BCVA outcomes for ranibizumab treated eyes were superior to triamcinolone in phakic eyes. However, in eyes that were pseudophakic at baseline BCVA outcomes were similar. As might be expected, IOP increase was more common in the triamcinolone group. These findings and additional efficacy and safety results reported in clinical trials of intravitreal steroid therapy for DMO are summarised in Table 1.

\section{Real-world data}

Jonas et al. investigated the effect of IVTA in DMO in a clinical interventional case series of 38 eyes ( 31 patients). ${ }^{15}$ This study involved IVTA doses of 20-25 mg and the average follow up was 13.2 months. VA and IOP increased significantly $(p=0.003)$ from week 1 , reaching a plateau at 1 to 7 months and returning to baseline values 8-9 months post injection. This study is limited by significant loss to follow up beyond 6 months; furthermore, the effects of IOP rise may be underestimated as there was no indication of how many of the participants were started on treatment. A subsequent retrospective comparative study of 22 eyes (19 patients) where patients received a second IVTA injection on average 10 months after the first, showed a significant improvement in BCVA by $1.8 \pm 2.1$ Snellen lines. ${ }^{16}$ IOP was found to increase significantly post injection, returning to baseline values approximately 6-8 months after. After the first injection, $41 \%$ of patients developed an IOP $>21 \mathrm{mmHg}$, $54 \%$ after the second injection and 50\% after the third injection. Later studies identified VA gains could be achieved with much lower doses $\left(4 \mathrm{mg}\right.$ ) of triamcinolone, potentially reducing the side-effect profile. ${ }^{17}$

Chan et al. carried out an early retrospective observational case series of IVTA in DMO. ${ }^{18}$ They observed 10 patients (10 eyes) who received an initial dose of IVTA followed by a subsequent dose after 26 weeks. After the first injection, BCVA improved significantly at 2, 4 and 9 weeks, compared to baseline. However, after the second injection, no significant BCVA improvement was observed at any time point. Significant CMT improvements were seen at 2, 4 and 9 weeks after the first and second injections compared to pre-injection values. In both cases optimal reduction in CMT was at 4 weeks. None of the eyes had an increase in IOP >21 mmHg after their first injection, but two eyes did after their second injection and both were controlled with topical therapy. Cataract progression was noted at 26 weeks. The authors proposed that the weak correlation between reduction in CMT and improvement in BCVA could be attributable to diabetic macular ischaemia. The weak correlation between reduction in CMT and BCVA improvement after IVTA injection has been described by Larsson et al. in a prospective, non-randomised clinical trial. ${ }^{19}$

Smithen et al. carried out a retrospective interventional case series involving 89 patients to assess the effect of 4 mg IVTA on IOP. ${ }^{17}$ Mean baseline IOP was $14.9 \mathrm{mmHg}$ with a mean increase of $8 \mathrm{mmHg}$. 36 patients (40.4\%) experienced a raised IOP (defined as a pressure of $\geq 24 \mathrm{mmHg}$ ) at a mean of 100.6 days (SD 83.1 days) post-treatment. Patients with a baseline IOP of $\geq 15 \mathrm{mmHg}$ were more likely to develop an IOP rise (relative risk $=2.1, p<0.01$ ). There were 32 patients $(36.0 \%)$ that received repeat injections, and there was no difference in the incidence of IOP elevation in those receiving multiple injections compared to single injection. The study is limited by a relatively short follow up period (mean duration of 9.2 months).

Kenacort 40 (Bristol-Myers Squibb, Australia) has been used as an offlabel intravitreal triamcinolone preparation, however there are reports that the preservative it contains may lead to incidences of sterile endophthalmitis. Stepien et al. performed a retrospective multicentre interventional case series where patients receiving IVTA with and without preservative were assessed for sterile endophthalmitis. ${ }^{20}$ Transition from preservative containing IVTA to preservative free IVTA resulted in a significant decrease in the incidence of sterile endophthalmitis. This has 


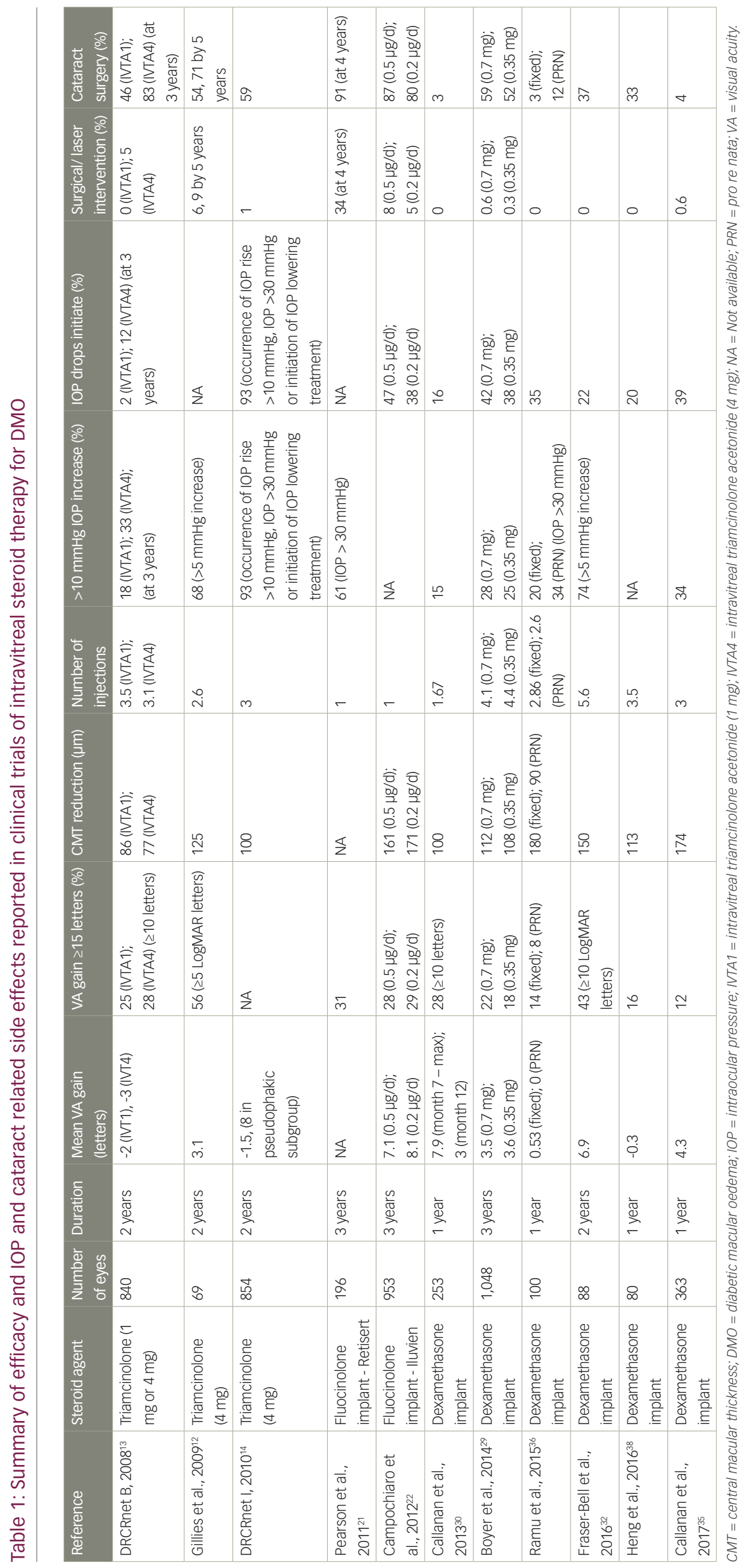


resulted in the development of preservative-free licensed triamcinolone preparations such as Trivaris and Triesence ${ }^{\circledR}$ (Alcon, Texas, US).

\section{Fluocinolone \\ Clinical trials}

Fluocinolone acetonide was initially delivered as the fluocinolone acetonide intravitreal implant (Retisert ${ }^{\circledR}$; Bausch \& Lomb, New York, US) - a non-biodegradable implant that required surgical placement via a pars plana incision and scleral suture. The implant released sustained concentrations of fluocinolone over 3 years. A prospective randomised controlled trial by Pearson et al. compared the Retisert implant $(n=127)$ with macular laser $(n=69) .{ }^{21}$ At 6 months follow up, the proportion of eyes that gained $\geq 3$ lines of vision was significantly higher in the Retisert group with $16.8 \%$ compared to the laser group with $1.4 \%$. At 2 years this percentage was $31.8 \%$ for the Retisert group and $9.3 \%$ for the laser group. Finally, at 3 years this percentage remained relatively stable in the Retisert group at $31.1 \%$ and rose to $20.0 \%$ in the laser group and at this endpoint the difference between the groups was not statistically significant. Despite these results, Retisert has not been approved for use in DMO due to a high rate of ocular complications. At 4 years $33.8 \%$ of eyes underwent glaucoma surgery and $2.4 \%$ of patients had to have their implant removed as a result of raised IOP. In addition, 91.0\% of phakic eyes needed cataract surgery within 4 years.

The FAME (Fluocinolone Acetonide in patients with diabetic Macular Edema) studies assessed 953 eyes of patients with persistent DMO after one or more laser therapy treatments. ${ }^{22}$ Patients were randomised in a 1:2:2 ratio between sham injection ( $n=185), 0.2 \mu \mathrm{g} / \mathrm{d}$ Iluvien ${ }^{\circledR}$ implant (Alimera Sciences, Georgia, US) ( $n=375)$, or $0.5 \mu \mathrm{g} / \mathrm{d}$ Iluvien implant $(n=393)$. At 36 months, $27.8 \%(0.5 \mu \mathrm{g} / \mathrm{d})$ and $28.7 \%(0.2 \mu \mathrm{g} / \mathrm{d})$ of all Iluvien treated eyes showed $a \geq 15$ letter improvement compared to $18.9 \%$ of sham eyes $(p=0.018)$. Subgroup analysis also showed a significant benefit in patients with chronic DMO (disease duration $>3$ years) treated with Iluvien ( $\geq 15$ letter improvement in $34 \%$ of patients treated with $0.2 \mu \mathrm{g} / \mathrm{d}$ and $28.8 \%$ receiving $0.5 \mu \mathrm{g} / \mathrm{d}$ dose compared to $13.4 \%$ in the sham group). In terms of adverse events, $80.0 \%$ of patients underwent cataract surgery. Furthermore, IOP lowering drops had to be initiated in $38.4 \%$ of those receiving the lluvien implant with a further $4.8 \%$ requiring an incisional IOP lowering procedure in the $0.2 \mu \mathrm{g} / \mathrm{d}$ group that was eventually licensed.

\section{Real-world data}

Alfaqawi et al. carried out a 12-month retrospective study of 28 patients receiving lluvien for refractory DMO. ${ }^{23}$ Improvement in BCVA was most marked (+16 letters) in eyes with relatively poor baseline VA because of chronic DMO ( $\leq 35$ letters), compared to those with 'relatively good' baseline VA who achieved the least VA gain. The mean baseline CRT was $494 \mu \mathrm{m}$ (SD 191); this reduced to a mean of $296 \mu \mathrm{m}$ at 12 months where CRT reduction was evident in $86 \%$ of eyes. In terms of adverse events, IOP increases were observed but no patient required IOP lowering surgery within the first year. This study also included two vitrectomised eyes, the authors note that although this number is too small to draw any definitive conclusions, these eyes had a long lasting reduction in CRT without the requirement for any rescue therapy. The results of this real-world study in terms of functional and anatomical outcomes are comparable to the FAME study. However, the study is limited by small numbers, its retrospective nature and absence of a control group. Schmit-Eilenberger also published a study of 15 eyes treated with Iluvien for refractory DMO. ${ }^{24}$ They observed $a+0.19 \pm 0.24$ LogMAR mean VA gain at the last follow-up visit, which was independent of prior treatments
Mean baseline CMT also decreased at the last follow-up visit (Table 2) and the mean IOP rise was $4.1 \pm 5.8 \mathrm{mmHg}$. There was a variable and short duration of follow-up (2-37 weeks).

Elaraoud et al..$^{25}$ undertook a retrospective, multicentre observational study of patients treated with Iluvien based on UK NICE guidelines. ${ }^{26}$ Participants were therefore pseudophakic and the majority had prior anti-VEGF and laser treatment. The mean 3 month BCVA and CMT change from baseline was +6.4 ETDRS letters ( $\mathrm{SD} \pm 7.2$ ) and $-148.9 \mu \mathrm{m}$ (SD \pm 240.6 ), respectively. In the five eyes that had undergone prior vitrectomy, the mean VA improved by +7.2 letters, and the mean CMT reduced by $176.8 \mu \mathrm{m}$. Although this subgroup is very small, this represents an area for potential further analysis. Although based across three sites, the number of patients in the study was still relatively low indicating the high threshold for the use of Iluvien. Furthermore, measures of efficacy and safety were limited by the short study duration.

Figueira et al. carried out a prospective non-randomised, open-label, phase IIII trial conducted across four sites in Portugal, designed to assess the efficacy and safety of lluvein in chronic DMO patients $(n=12)$ who were insufficiently responsive to other therapies. ${ }^{27}$ The study involved eight pseudophakic and four phakic eyes and mean baseline BCVA and CMT was similar for both groups. At 12 months, mean BCVA increased by +6.75 ETDRS letters in pseudophakic eyes and decreased by 2.5 ETDRS letters in phakic eyes most likely due to cataract formation. Mean CMT decreased in both subgroups. Mean IOP increased by $2.58 \mathrm{mmHg}$ in all eyes and the increase was managed with IOP lowering drops in all patients. Although this study provides some insight into the differences that can be expected between phakic and pseudophakic patients, it is limited by its small sample size.

The Iluvien Registry Safety Study (IRISS) registry is an open label, registry study of 231 eyes designed to assess the real-world tolerability of Iluvien across multiple European countries where it is currently licensed. ${ }^{28}$ Encouragingly, mean VA increased by +4.4 and +5.9 letters at 3 and 12 months respectively and mean IOP by approximately $+4 \mathrm{mmHg}$ at 12 months. A high percentage of participants were pseudophakic (due to the fact that the UK was the largest enrolling country). There was no control group in the IRISS study, and hence other differences (not just lens status) between the two study populations, could have also contributed to this variation. The IRISS real-world study reports a lower rate of incisional glaucoma drainage surgery (0.8\%) than the FAME study where $4.8 \%$ of study eyes required incisional drainage surgery to control IOP. However, the follow-up duration of the IRISS study is 12 months, whereas the $4.8 \%$ figure quoted from the FAME study represents the proportion of patients requiring an incisional glaucoma procedure over 36 months. The percentage of patients in the FAME study requiring an incisional procedure at 12 months was also $0.8 \%$.

\section{Dexamethasone implants}

\section{Clinical trials}

Boyer et al. examined the safety and efficacy of the Ozurdex ${ }^{\circledR}$ (Allergan, New Jersey, US) dexamethasone intravitreal implant in DMO over a 3-year period in the MEAD (Macular Edema: Assessment of Implantable Dexamethasone in Diabetes) study. ${ }^{29}$ The study comprised two parallel randomised, multicentre, masked phase III controlled trials involving 1,048 patients with DMO. Inclusion criteria were BCVA of 20/50 to 20/200, and optical coherence tomography (OCT) measured CMT of $\geq 300 \mu \mathrm{m}$. Patients were randomised equally to receive dexamethasone implant $0.7 \mathrm{mg}$, dexamethasone implant $0.35 \mathrm{mg}$ or sham treatment. Patients could be retreated no more often than every 6 months. Results showed 


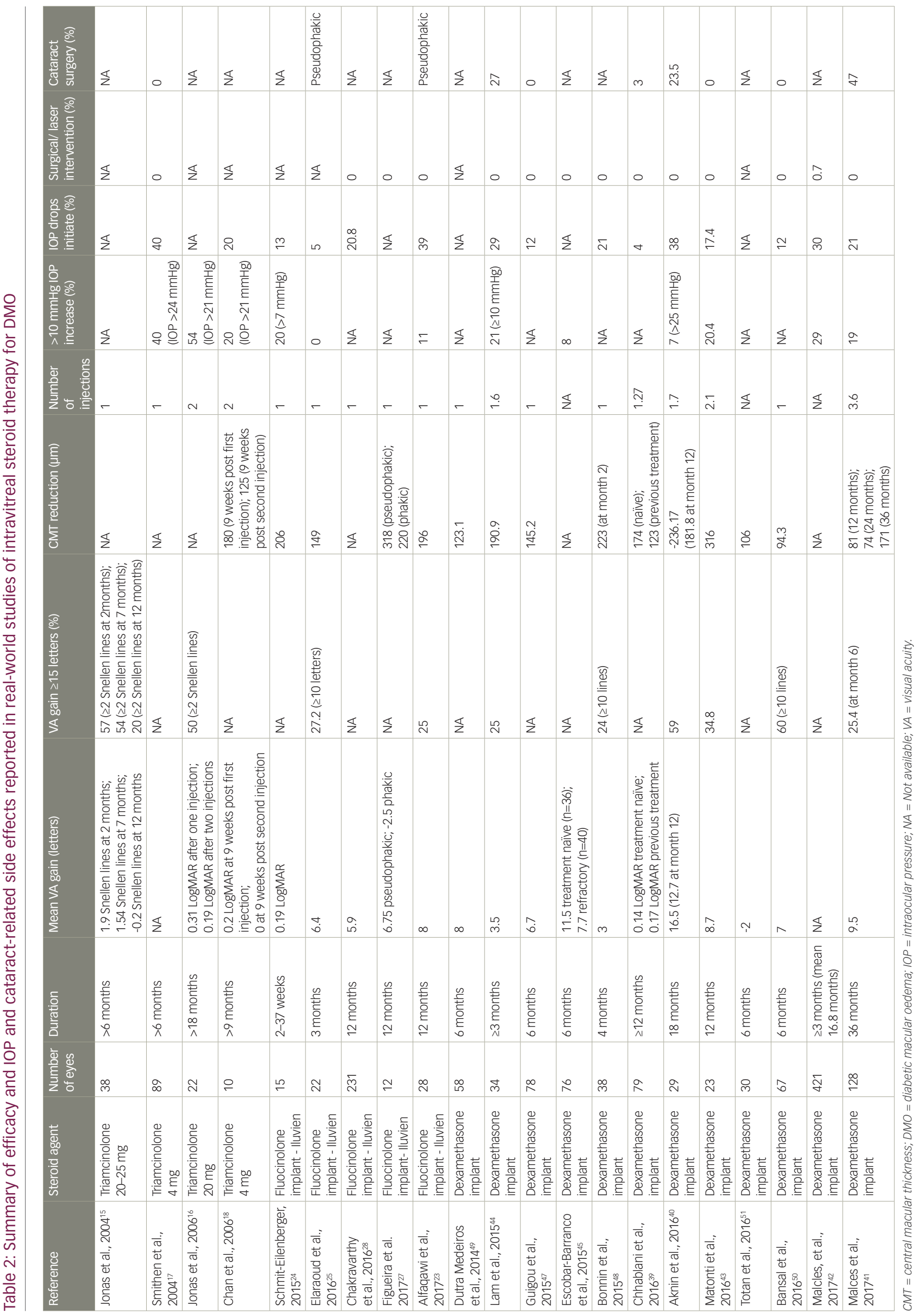


that a significantly higher proportion of patients receiving steroid treatment achieved a 15 letter BCVA gain $(22.2 \%$ in the $0.7 \mathrm{mg}$ group and $18.4 \%$ of the $0.35 \mathrm{mg}$ group), compared with the sham group (12.0\%, $\mathrm{p} \leq 0.018)$. Furthermore, the mean reduction in CMT from baseline, was also greater in the groups receiving dexamethasone implants at a dose of $0.7 \mathrm{mg}(-111.6 \mu \mathrm{m})$ and $0.35 \mathrm{mg}(-107.9 \mu \mathrm{m})$ versus sham $(-41.9 \mu \mathrm{m}$; $\mathrm{p}<0.001)$. IOP rise $\geq 10 \mathrm{mmHg}$ from baseline at any study visit was $27.7 \%$, $24.8 \%$ and $3.7 \%$ in the $0.7 \mathrm{mg}, 0.35 \mathrm{mg}$ and sham groups, respectively. Two patients $(0.6 \%)$ in the $0.7 \mathrm{mg}$ group and one patient $(0.3 \%)$ in the $0.35 \mathrm{mg}$ group required trabeculectomy surgery. The rate of cataract surgery during the 3-year study was $59.2 \%, 52.3 \%$ and $7.2 \%$ in the 0.7 $\mathrm{mg}, 0.35 \mathrm{mg}$ and sham groups, respectively. Loss of effectiveness of the dexamethasone implant became apparent before the minimum 6-month interval for retreatment that was prospectively chosen in the trial. To ensure the drug was on board, the primary endpoint was changed from 36 to 39 months. A limitation of the study is the significant loss to follow up, including over a third of patients in the dexamethasone treatment groups and over half of patients in the sham group exiting the study early.

The PLACID study is a randomised control trial where patients with diffuse DMO were allocated to receive a $0.7 \mathrm{mg}$ dexamethasone implant followed by laser photocoagulation at 1 month $(n=126)$, or to sham injection followed by laser photocoagulation at 1 month $(n=127) .{ }^{30}$ Subjects were eligible for an additional dexamethasone implant or sham injection 6 months after the first injection and further macular laser as needed up to every 3 months. At month 12 there was no significant difference $(p=0.31)$ in the percentage of patients achieving at least a 10-letter improvement in BCVA between the groups. However, there was a significantly greater improvement in BCVA from baseline to various time points up to 9 months in the dexamethasone group which is also supported by the area under the curve analysis for BCVA over the 12 months, which showed a significant benefit for these patients compared with macular laser alone. In the dexamethasone group IOP lowering medication was commenced in $15.9 \%$ of patients, compared with $1.6 \%$ in the laser alone group. No incisional glaucoma surgery was necessary in either group. Cataract surgery was performed in 3.2\% of eyes treated with the dexamethasone implant plus laser over the 12 months of this study compared to $3.9 \%$ in the laser alone group. The therapeutic effect of the dexamethasone intravitreal implant is around 4 months However, the mistaken belief that it lasts 6 months in all patients has led to some industry sponsored clinical trials having difficulty meeting their originally specified endpoints.

Gillies et al. reported the 12-month results of a randomised clinical trial comparing bevacizumab with dexamethasone intravitreal implant in the BEVORDEX study. ${ }^{31}$ There were 88 eyes (61 patients) with centre-involving DMO were randomised to receive bevacizumab intravitreal injections (42 eyes) up to 4-weekly or to receive a dexamethasone implant up to every 16 weeks (46 eyes), both treatments were given as required (pro re nata; PRN). In $40.0 \%$ of the bevacizumab group and $41 \%$ of the dexamethasone group there was an improvement in BCVA by at least 10 IogMAR letters, achieved with a mean of 8.6 injections and 2.7 injections for the bevacizumab and dexamethasone groups, respectively, over 12 months. In the pseudophakic subgroup, bevacizumab treated eyes gained a mean of 7.7 letters and dexamethasone treated eyes gained a mean of 10.4 letters, but this difference was not statistically significant. There were $11.0 \%$ of the dexamethasone group who lost $>10$ letters at one year compared with none in the bevacizumab group. Most of this was deemed to be the result of increased cataract and the remaining patient who had decreased vision had undiagnosed syphilis and developed chorioretinitis post Ozurdex injection. An increase in cataract density by $\geq 2$ grades was observed in $13.0 \%$ of eyes in the dexamethasone group and in $4.8 \%$ of eyes in the bevacizumab group at 12 months. Additionally, $26 \%$ of eyes in the dexamethasone group had an IOP of $>25 \mathrm{mmHg}$ within the first year none of which required incisional glaucoma surgery. The Impact of vision questionnaire was also completed by participants as a qualitative measure of the different treatments. There were 25 of 34 patients who had one eye enrolled in the study completed the questionnaire at baseline and 12 months, no significant difference was found between the mean improvements in scores between the two groups ( $p>0.1$ ). Twenty-five out of 27 patients had both eyes enrolled in the study, and responded to which treatment type they preferred; $33.0 \%$ chose bevacizumab, $46.0 \%$ chose dexamethasone, and $21.0 \%$ had no preference $(p>0.1)$. No significant difference in patient reported outcomes between the two treatments was noted at 12 months; although, it should be noted patients attended every 4 weeks for follow-up in the clinical trial independent of treatment allocation.

One of the main benefits of the BEVORDEX trial was the 16-week minimal retreatment interval compared with the 6 months chosen in MEAD, resulting in decreased recurrence of oedema. ${ }^{31}$ However, this effect needs to be balanced with the potential for increased rates of cataract formation and IOP rise and hence the authors do not recommend a shorter interval than 16 weeks.

Of the initial 88 eyes from 61 patients, 68 eyes from 47 patients completed 24 months of follow-up. ${ }^{32}$ There was an equal number of loss to follow-up from each group (10 eyes). There were $45 \%$ of bevacizumab treated eyes and $43 \%$ of dexamethasone treated eyes that maintained a $>10$ letter improvement from baseline at 24 months. At the same endpoint, $2 \%$ of bevacizumab treated eyes and $11 \%$ of dexamethasone treated eyes had a VA decrease $\geq 10$ letters. There was no significant difference between bevacizumab and dexamethasone treated eyes in terms of visual gain at 24 months, particularly for pseudophakic eyes. It is likely a larger study would have identified greater gain in the bevacizumab group for phakic eyes. In terms of number of injections in year 2 , the bevacizumab group received a mean of 4.8 (SD 5.1) compared to 2.2 (SD 1.2) in the dexamethasone group.

Interestingly, a post-hoc analysis looking at changes in vessel calibre identified a significant reduction in venular diameter over the study in dexamethasone treated eyes not seen with bevacizumab treated eyes. ${ }^{33}$ Additionally, a post-hoc analysis identified more rapid regression of hard exudates in dexamethasone treated eyes compared with bevacizumab treated eyes. ${ }^{34}$ These observations suggest the two drugs have different mechanisms of action.

Callanan et al. carried out a 12-month multicentre, open-label randomised controlled trial of 363 patients comparing the safety and efficacy of the dexamethasone implant against ranibizumab in patients with $\mathrm{DMO}^{35}$ The dexamethasone implant was given at baseline, 5 and 10 months, and ranibizumab was given monthly until maximum VA was achieved (three consecutive stable measurements), injections could then be suspended but reinitiated if a decrease in BCVA occurred. The mean average BCVA gain over 12 months was 4.34 letters with dexamethasone (4.60 letters in the pseudophakic subgroup) and 7.60 letters with ranibizumab. The lower limit of the $95.0 \%$ confidence interval $(\mathrm{Cl})$ of the betweengroup difference was -4.74 letters, and therefore, dexamethasone implant was demonstrated to be noninferior to ranibizumab based on the prespecified noninferiority margin of 5 letters. Between-group differences in change from baseline CMT favoured dexamethasone at $1,2,6$ and 7 months $(p \leq 0.007)$ and ranibizumab at 4, 5, 9 and 10 months $(p<0.001)$. This cyclical pattern of CMT reduction is a likely manifestation 
of the dexamethasone implant efficacy wearing off at 5 months; this is in contrast to the slower, sustained and progressive decrease in CMT seen in the ranibizumab group.

IOP-related adverse events were reported in $43.1 \%$ of patients in the dexamethasone group compared with $6.0 \%$ of patients in the ranibizumab group $(p<0.001)$. There were $39.2 \%$ of patients in the dexamethasone group requiring IOP-lowering medication in the study eye (19.3\% required one medication, $7.2 \%$ required two medications and $12.7 \%$ required three or more medications). In those with increased IOP, the IOP decreased by 4 months after Ozurdex injection. Seven patients in the dexamethasone group, and one patient in the ranibizumab group, underwent cataract surgery in the study eye.

The median number of study treatments administered over the course of 12 months was three in the dexamethasone group, compared with nine in the ranibizumab group. Patients with DMO often have additional co-morbidities requiring visits to various healthcare providers and hence fewer visits for the treatment of DMO can help relieve the burden on the patients and their carers. ${ }^{10}$

This is the first trial to compare the dexamethasone implant with a licensed anti-VEGF (ranibizumab) as opposed to previous comparisons with bevacizumab in the BEVORDEX trial. ${ }^{31}$ The study also benefits from a large sample size ( $n=363$ study eyes). Cataract associated with the dexamethasone implant was one of the main adverse events associated with treatment. It would be beneficial to note the impact this has on visual outcomes if the study was continued for a longer duration. In addition, the 5-month treatment intervals are likely to be suboptimal, as the duration of action of the DEX implant is probably closer to 4 months in some patients. It is also worth noting that the patients in this study had relatively chronic DMO (mean duration 2.5 years in the ranibizumab group and 3 years in dexamethasone group).

The OZDRY study by Ramu et al. is a 12-month, prospective, randomised, active controlled, non-inferiority trial comparing fixed 5-month interval dexamethasone implant with PRN dexamethasone implant based on pre-defined retreatment criteria. ${ }^{36}$ There were 100 patients recruited into the study and randomised evenly between the two groups. The non-inferiority margin was pre-defined as $<5$ ETDRS letters difference. The intention to treat (ITT) analysis effect estimate was $-0.34(-5.49$, 4.81), which overlapped the non-inferiority margin by 0.49 letters. Per protocol and post-hoc ITT sensitivity analysis based on last observation carried forward did, however, demonstrate non-inferiority $(90 \% \mathrm{Cl})$. With regard to secondary outcome measures, BCVA gain of $\geq 15$ letters was observed in $14 \%$ of fixed dose patients and $8 \%$ of PRN patients (odds ratio 1.3), mean CMT change from baseline was -180 and -90 from a baseline of 293 and 372 in the fixed and PRN groups, respectively. In terms of safety outcomes, IOP rise of $>30 \mathrm{mmHg}$ was observed in $20 \%$ and $34 \%$ of patients in the fixed and PRN groups, respectively, and of these patients, 64\% were started on topical IOP lowering medications. No patient required IOP lowering surgery. There were $79 \%$ of phakic patients in the fixed arm who were observed to have progression of cataract by at least one grade according to the Lens Opacities Classification system II grading score. This figure was similar (77\%) in the PRN group. The 12-month endpoint of the study may have benefited the fixed group more so than the PRN group as all patients would have received a 10-month injection from which the maximal effect on vision and macular thickness would be expected at 12 months, which will not have been the case in the PRN group. Although this did not alter the visual outcome between arms, a longer study duration may mitigate the effect this may have had on the CMT differences between the two groups. The development of cataract makes it difficult to assess the impact of treatment on diabetic maculopathy. Study design could have been improved by either having an entry requirement of pseudophakic eyes or by increasing study duration to 2-3 years to assess eyes in the long term when cataract had been addressed. The same study group also published patient reported outcome measures from both groups. ${ }^{37}$ There was a significant improvement in satisfaction, as measured with the Retinopathy Treatment Satisfaction Questionnaire from baseline to 1 year but no significant difference between the two groups.

This study by Heng et al. randomised 80 patients into two groups, one receiving combined dexamethasone and MLT and the other group MLT alone. ${ }^{38}$ Dexamethasone was given on a PRN basis after a baseline and 16-week injection and MLT was given PRN at 16 week intervals. At the 12-month endpoint, the mean change in BCVA was -0.3 (SD 11.4) ETDRS letters in the combination group and +0.4 (SD 9.6) ETDRS letters in the MLT arm. Mean CMT change at 12 months was $-113 \mu \mathrm{m}$ in the combination arm and only $-17 \mu \mathrm{m}$ in the MLT arm $(p<0.001)$. Raised IOP requiring topical treatment was observed in $20 \%$ of eyes in the combination group, and $2.5 \%$ in the MLT group. Thirty-three percent of phakic patients in the combination group required cataract surgery. These results demonstrate no significant difference between the groups in terms of BCVA but a significantly higher reduction in CMT in the combination group. These BCVA findings contradict the results of the MEAD ${ }^{29}$ and PLACID ${ }^{30}$ trials despite more frequent dosing, there is however a more uniform decrease in CMT compared to a more fluctuating pattern observed in MEAD and PLACID. This suggests that although the anatomical benefits of more frequent dosing were evident, the functional benefits were confounded likely by cataract formation. The authors of the study suggested that there may be a ceiling effect in VA gain as the inclusion criteria for baseline BCVA was between 54 and 78 ETDRS letters.

\section{Real-world data}

Chhablani et al. carried out a 12-month retrospective, multicentre, interventional case series of 79 eyes with DMO treated with dexamethasone implant. ${ }^{39}$ Improvements in BCVA were observed in a treatment naive subgroup of patients, where the mean baseline value of $0.58 \pm 0.25 \log M A R$ improved to $0.44 \pm 0.33(p=0.05) \log M A R$ at last follow-up and in the previously treated subgroup where BCVA improved from $0.65 \pm 0.34 \log$ MAR at baseline to $0.48 \pm 0.35 \log$ MAR at last followup. There was also a significant improvement in CMT in both groups $(550.64 \pm 130.07 \mu \mathrm{m}$ to $377.1 \pm 105.8 \mu \mathrm{m}$ in naive eyes and $535.3 \pm 196.9 \mu \mathrm{m}$ to $413.0 \pm 242.4 \mu \mathrm{m}$ in previously treated eyes). The mean treatment free interval was $10.53 \pm 7.8$ months for treatment naïve and $6.5 \pm 4.5$ months for previously treated patients. Only $17.7 \%$ of eyes received more than one Ozurdex injection and $72.0 \%$ did not require any additional treatment during the mean follow-up period of $18.3 \pm 6.1$ months. Six eyes (7.6\%) had significant IOP rise which was controlled with topical anti-glaucoma medications, which is lower than most other studies. This study benefits from a relatively large sample size (79 eyes). The main limitation is the retrospective nature and non-standardised criteria for retreatment.

Aknin et al. undertook a retrospective non-comparative study of 29 patients who received dexamethasone implant for $\mathrm{DMO} .{ }^{40}$ Significant VA improvement was observed with a mean gain of 12.7 letters at month 12, and 16.5 letters at month 18 . Significant CMT reduction was observed from day 7 , with a mean decrease of $181.8 \mu \mathrm{m}$ at month $12(p<0.0001)$ and $236.17 \mu \mathrm{m}$ at month $18(p=0.0313)$. There were 15 patients who received one injection, 10 patients received two injections, three patients received three injections and one patient received four 
injections, over the 18-month study duration. The mean interval between dexamethasone implant re-injection was 5.6 months. In terms of adverse events, topical IOP lowering treatment was initiated in $38.0 \%$ of patients, and $23.5 \%$ of phakic patients needed cataract surgery during follow-up Limitations of this study include the fact that only 16/29 patients had 12-month data and 6/29 had 18-month data.

Malcles et al. carried out a retrospective study of 128 eyes of 89 patients with DMO treated with dexamethasone implant across two sites in France. ${ }^{41}$ The average follow-up duration was 16 months (range 1-40 months). The mean number of injections per patient was 3.6 and the mean interval to dexamethasone implant re-injection was 7.3 months with the number of injections appearing to decrease over time. There was a mean BCVA gain of 9.5 letters at month 36 and CMT decrease from $451 \mu \mathrm{m}$ at baseline, to $370 \mu \mathrm{m}$ at month $12(\mathrm{p}=0.001), 377 \mu \mathrm{m}$ at month $24(p=0.004)$, and $280 \mu \mathrm{m}$ at month $36(p=0.001)$. The mean number of injections received by patients was $3.6(95 \% \mathrm{Cl}, 3-4)$, and the mean time to retreatment was 7.3 months $(95 \% \mathrm{Cl}, 6.5-8.1)$ over the 36 months. IOP $\geq 25 \mathrm{mmHg}$ was seen in $10.2 \%$ of eyes and IOP lowering topical treatment was initiated in $21.0 \%$. There was no demonstrable cumulative effect on IOP due to repeat injections. Cataract surgery was performed in $47.0 \%$ of phakic eyes and retinal neovascularisation was observed in two cases during follow-up. This is a useful study due to the large number of patients and relatively long duration of follow-up. However, limitations include retrospective data collection and lack of a control group. Furthermore, follow-up periods and criteria for retreatment were not standardised.

The Safety of Intravitreal dexamethasone implant (SAFODEX) study is a retrospective study designed specifically to analyse the incidence, risk factors and timings of IOP elevation with dexamethasone implant. ${ }^{42}$ Indications other than DMO were included and ocular hypertension $(\mathrm{OHT})$ was defined as IOP $\geq 25 \mathrm{mmHg}$ or an increase of $\geq 10 \mathrm{mmHg}$ from baseline. The mean follow-up duration was 16.8 months (3-55), over this time OHT was recorded for $28.5 \%$ of eyes $(20.0 \%$ had an IOP $\geq 25 \mathrm{mmHg}$ and $6.0 \% \geq 35 \mathrm{mmHg}$ ) and IOP lowering medication was initiated in $31.0 \%$ of eyes (topical IOP lowering treatment was sufficient in $97.0 \%$ of these cases). Glaucoma filtration surgery was required in three eyes (0.7\%). Al of these patients had established glaucoma and were treated with either dual $(n=2)$ or triple topical therapy $(n=1)$ at baseline. In 24 cases $(6.0 \%)$, dexamethasone treatment had to be stopped due to poor tolerance of raised IOP. The risk of IOP elevation was not increased in those who had early retreatment (between 3-6 months). Multivariate analysis identified six main risk factors for dexamethasone implant-associated IOP rise: Age $\leq 60$, male gender, retinal vein occlusion, uveitis, pre-existing glaucoma on dual or triple therapy and type 1 diabetes ( $p<0.05$ for all the above). The main limitation of the study is its retrospective nature meaning that there were irregular intervals between IOP measurements. Furthermore, the criteria for initiating IOP lowering treatment was not pre-specified. This study does, however, provide an insight into timings and risk factors of one of the main adverse effects (IOP), limiting the use dexamethasone implant and serves as a foundation for further studies. Interestingly, the peak IOP rise after delivery of the dexamethasone implant was at 8 weeks which has implications for when clinicians should arrange the initial IOP check.

Matonti et al. also carried out a retrospective, noncomparative study of 23 patients who received dexamethasone intravitreal implant for treatment of $\mathrm{DMO}^{43}$ There were three patients who received three dexamethasone implant injections over 12 months with the rest (20) receiving two injections. The mean interval for reinjection was 5.4 months. BCVA increased by 10.3 letters and peaked at month 1; at 12 months BCVA improvement was 8.7 letters. Mean CMT reduction at month 12 was $316 \mu \mathrm{m}$. In terms of IOP, increase $\geq 25 \mathrm{mmHg}$ was seen in $11.7 \%$ patients (maximum at month 2). Topical treatment was initiated in $17.4 \%$ patients at month 2 . No cataract surgery was needed in patients during the 12 months, although it is important to note that a longer follow up would likely reveal a higher rate. Vitreous haemorrhage associated with injection was seen in two patients with no associated retinal complications.

The main limitation of this study is the small sample size. Patients who received a second injection before month 5 had a 9.9 letter greater improvement at month $12(p=0.17)$ compared to patients retreated at $\geq 5$ months, baseline difference between these two subgroups was 0.6 letters. The earlier re-treated group received an average of 2.16 injections in 1 year while the second group received a mean of 2.08 dexamethasone implant injections in the same period. This study suggests that early retreatment may stabilise macular oedema more effectively resulting in less retreatment thereafter, although further work is required to test this hypothesis.

The Chart Review of Ozurdex ${ }^{\circledR}$ in Macular Edema (CHROME) study by Lam et al. was a retrospective cohort study of patients with macular oedema secondary to retinal disease treated with dexamethasone intravitreal implant. ${ }^{44}$ Of the 120 study eyes, 34 had DMO and the minimum followup duration was 3 months. The mean peak improvement in BCVA lines of vision in study eyes with DMO was $0.7 \pm 0.5$ (non-significant, $p>0.05$ ), a statistically significant peak mean gain of $1.4 \pm 0.5$ lines $(p<0.05)$ was observed in the pseudophakic DMO subgroup, suggesting confounding of VA gains by cataract progression. Significant decreases in CMT were observed $(-190.9 \pm 23.5 \mu \mathrm{m})$. IOP increases of $\geq 10 \mathrm{mmHg}$ occurred in $20.6 \%$ of DMO eyes and IOP-lowering medication was initiated in $29.4 \%$, half of which required dual therapy and over a third required triple therapy. Cataract surgery was performed in $27.3 \%$ of phakic eyes.

Escobar-Barranco et al. carried out a prospective study of 76 patients (40 refractory and 36 naïve) who had received a dexamethasone implant (+/- photocoagulation). ${ }^{45}$ Patients were followed up monthly for a total duration of 6 months. BCVA change in the treatment naive group was +11.5 letters compared with a gain of 7.7 letters in the refractory group at the 6-month endpoint. CMT decreased significantly in both groups $(p<0.001)$. There were six patients who developed a transient IOP increase of $>10 \mathrm{mmHg}$ (four in the naïve group and two in the refractory group).

The Multicenter Ozurdex Assessment for Diabetic Macular Edema: MOZART study by Guigou et al. is a retrospective study of 74 patients who were followed up for a minimum of 6 months (mean follow-up: 9.8 months). ${ }^{46}$ At month 6 the mean change in BCVA and CMT was +7.6 letters and $-135 \mu \mathrm{m}$ respectively with a better BCVA gain in treatment naive patients $(p<0.05)$. These VA results correlate with the findings of Escobar-Barranco et al. and both sets of authors suggest that dexamethasone intravitreal implant should be considered earlier in the disease process. ${ }^{45}$

Guigou et al. carried out another similar 6-month retrospective multicentre noncomparative study of 78 patients with DMO (78 eyes). ${ }^{47}$ BCVA, CMT, IOP and cataract progression were studied at baseline, 1, 2, 4 and 6 months. The mean 6-month improvement in BCVA and decrease in CMT was 6.7 letters and $145.2 \mu \mathrm{m}$ respectively. IOP rise was observed in $11.7 \%$ of patients, all of whom were managed with topical treatment. No glaucoma or cataract surgery was necessary and no endophthalmitis was reported. 
The following studies by Bonnin et al. ${ }^{48}$ Dutra Medeiros et al., ${ }^{49}$ Bansal et al..$^{50}$ and Totan et al. ${ }^{51}$ are summarised in Table 2. They are all similar studies with relatively short (4-6 month) follow-up. As might be expected most studies found a significant improvement in BCVA and CMT, the exception was in the study by Totan et al. where BCVA significantly improved at 1 and 3 months but not at 6 months where there was the equivalent of a 2 ETDRS letter loss..$^{51}$ The patient cohort in this study had not responded to previous anti-VEGF therapy and treatment may have been wearing off towards the end of the 6 months. The studies by Dutra Medeiros et al. ${ }^{49}$ and Bansal et al. ${ }^{50}$ specifically recruited patients with refractory/chronic DMO with encouraging results. With regards to adverse events, as might be expected IOP rises were observed, the majority of which were controlled with topical therapy or conservative management. Low rates of cataract surgery are likely to be a reflection of the short follow-up duration in these studies.

Khan et al. carried out a meta-analysis of 3,859 patients among 15 studies assessing the effect on BCVA and efficacy of dexamethasone implant for recalcitrant DMO. ${ }^{52}$ These included three randomised studies, six retrospective studies and six prospective observational studies. Using a random effects model, patients treated with dexamethasone implant for DMO refractory to anti-VEGF therapy (at least six prior anti-VEGF treatments) gained a mean of 20 ETDRS letters. All studies showed an improvement in BCVA. The mean follow-up period was 6 months (range 3-36). A funnel plot outlined five outlier studies, which may skew the mean results. The authors suggest that the findings of this analysis support the role of dexamethasone implant in the treatment of DMO refractory to anti-VEGF therapy. Due to the relatively short mean follow-up in the analysis, analysis of ongoing longer studies is needed to determine whether repeated injections can yield similar beneficial effects. The study is also limited by relatively high heterogeneity among the included studies (I2 value of 90.4).

\section{Anterior chamber migration of steroid implants}

A rare but potentially serious complication of intravitreal steroid implants is migration into the anterior chamber. Initial reports of anterior chamber migration were mainly in aphakic eyes and it was thought that this was the main risk factor. This would also provide an explanation as to why this complication wasn't observed in phase III clinical trials as these high-risk patients would have been excluded. Khurana et al. published a case series of 15 patients with 18 episodes of dexamethasone implant anterior chamber migration. ${ }^{53}$ Of these patients, six were aphakic, four had an anterior chamber intraocular lens, two had a scleral-fixated posterior chamber intraocular lens (PCIOL), two had a PCIOL and one had an irisfixated PCIOL. All patients had prior pars plana vitrectomy, and 93\% had no lens capsule. The mean time from injection of the dexamethasone implant to detection of migration was 13 days (range, 5-44 days). With regard to the sequelae of anterior chamber migration, 14 patients developed corneal oedema. In $71 \%$ of these patients, the oedema did not spontaneously resolve even after removal of the implant, and $43 \%$ ultimately required corneal transplantation. It was observed that earlier removal of the implant, following detection, reduced the likelihood of chronic corneal oedema.

The stable pharmacokinetics of steroid implants may be useful in the setting of vitrectomised eyes where intravitreal anti-VEGF therapy may leave the eye more quickly. However, steroid implants should be avoided if there is a communication between the posterior and anterior segments, for example a large peripheral iridectomy, zonular dehiscence or absent posterior capsule. A YAG capsulotomy in the setting of standard previous cataract surgery with the intraocular lens in the bag is not a contraindication to steroid implants.

\section{Guidelines}

The importance of modifying systemic risk factors such as blood pressure and glycaemic control should always be emphasised to patients as this can improve DMO. Multidisciplinary care should be utilised to support this endeavour, with encouragement of regular exercise and improved diet. The FIELD ${ }^{54}$ and ACCORD-EYE ${ }^{55}$ studies also suggest a beneficial role of fenofibrate on diabetic retinopathy with a mechanism of action that is independent of lipid control.

In the majority of patients, anti-VEGF agents would be the first-line treatment option in centre involving $\mathrm{DMO}$, taking into account their efficacy and favourable side-effect profile. In countries such as the UK, steroid usage in the context of DMO is restricted to patients who are pseudophakic or are due to have cataract surgery in the near future. Other than non-response to anti-VEGF treatment this factor should be one of the main considerations in commencing treatment with an intravitreal steroid agent. Ideally steroid therapy should be commenced either 2-4 weeks prior, or during, cataract surgery to prevent worsening macular oedema in the post-operative period.

Another indication, primarily for practice in the UK is for patients with a CMT of less than 400 microns, where the use of anti-VEGF agents of not licensed by The National Institute for Health and Care Excellence (NICE). Here, steroid therapy is a viable option.

As highlighted by some of the studies in this review, the pathophysiology of DMO involves complex molecular pathways which are not exclusively driven by VEGF. After 24 months of monthly intravitreal ranibizumab injections in the RISE study, $24 \%$ of study eyes had persistent macular oedema with CMT > 250 micrometers. In anti-VEGF non-responders, real-world studies support the efficacy of steroids in both functional outcomes of improving vision and anatomical outcomes in reducing oedema. ${ }^{52}$ However, there are a lack of good clinical trial data to guide clinicians as to when switching might be appropriate. Early analysis of data from DRCRnet Protocol I suggested long term visual outcomes at 3 years could be predicted at 12 weeks. However, few eyes that completed follow-up lost vision with long-term anti-VEGF therapy so the key will be defining eyes which have the potential for gain in vision by switching from antiVEGF to steroid agents.

Steroid implants may also be considered in vitrectomised eyes because of their stable pharmacokinetics. ${ }^{57}$ There may be reduced efficacy of intravitreal anti-VEGF agents in vitrectomised eyes because of increased drug clearance,58 however, Bressler et al. in a small subgroup analysis of DRCRnet Protocol I did not identify that the 25 vitrectomised eyes receiving ranibizumab had significantly worse visual outcomes than the rest of the cohort (325 eyes). ${ }^{59}$

Patient factors are another important consideration. The decision between a steroid agent and anti-VEGF treatment should be made on a case by case basis. An example of where a steroid agent may be useful over anti-VEGF might be when it would be difficult for a patient to attend frequently for repeat injections; although, additional visits to manage cataract and raised IOP should be considered. This is particularly important in diabetic patients who often have various other hospital appointments that they need to attend for other related co-morbidities. Real-world outcomes with intravitreal steroids seem to more closely mirror clinical trial outcomes than with intravitreal anti-VEGF agents which may reflect the difficulty in delivering frequent intravitreal procedures in clinical practice (Figure 1).56,60,61 
Finally, in a patient with a history of cardiac or cerebrovascular disease, steroid therapy can be considered due to the theoretical increased risk of potential arterial thromboembolic events with anti-VEGF therapy. A cochrane review found that adverse events such as death and systemic arterial thromboembolic events are unlikely in the short to medium time frame in populations included in clinical trials, however further data is required to assess this risk in high-risk patients. ${ }^{5}$ Most seminal clinical trials excluded patients with a thromboembolic event in the last 3-6 months. ${ }^{9,56,60,62}$

With regard to choosing between the different steroid agents, there is no head-to-head clinical trial to determine relative safety and efficacy. Table 2 identifies that IOP elevation is most predictable and least likely to require glaucoma drainage surgery with the Ozurdex dexamethasone implant, making it our preferred intravitreal steroid treatment for DMO. The fluocinolone implant could be considered if repeat procedures for implantation are likely to be difficult, such as in cases where sedation or a general anaesthetic is required. There is also evidence for the use of fluocinolone in chronic DMO although this is likely to be a class effect. Although triamcinolone is the cheapest of the intravitreal steroid agents it is associated with a poor IOP profile. Its use may be considered in cases where there is a communication between the anterior and posterior chambers such as posterior capsule tear or zonular dehiscence where there is a risk of the posterior segment implant migrating to the anterior chamber. It is preferable to use a licensed formulation of triamcinolone because of reported cases of sterile endophthalmitis with Kenalog.

Real-world efficacy and safety outcomes measures would benefit from being standardised in the same way as the International Centre for Healthcare Outcome Measures (ICHOM) has agreed a consensus
Figure 1: Comparison of 12 month mean ETDRS letter gain in registration clinical trials (blue) and real-world trials (red)

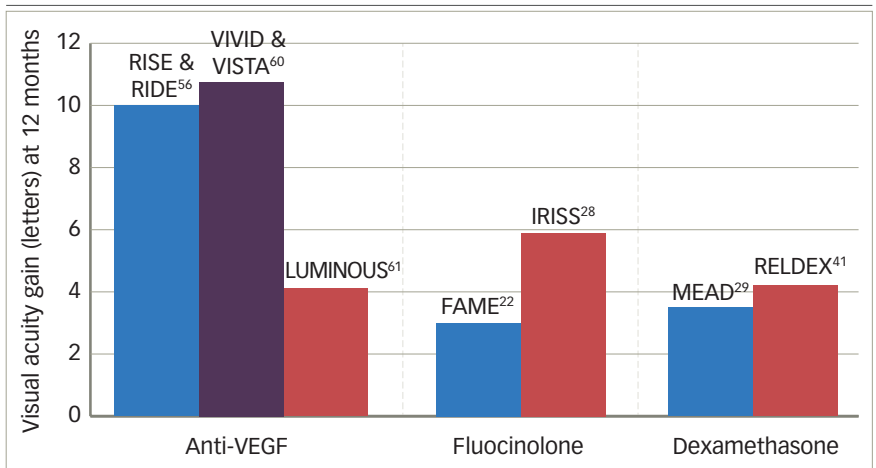

Baseline characteristics, including lens status, will be different between studies, and direct comparisons between visual acuity gains must be interpreted with caution; however, there is a clear trend for real-world data to be more comparable to registration clinical trial outcomes for steroid agents versus anti-VEGF agents in the management of centre-involving DMO. An explanation for this observation could be difficulty delivering frequent intravitreal therapy in the real-world setting. ETDRS = Early Treatment Diabetic Retinopathy Study; VEGF = vascular endothelial growth factor.

for neovascular AMD real-world data collection. Agreeing baseline characteristics to record, defining re-treatment criteria, measures of IOP elevation, criteria for initiating IOP lowering surgical intervention, defining cataract progression, quality of life indices, resource utilisation, visit numbers and systemic side-effects would allow long-term functional, anatomical and adverse effects of treatment to be pooled, and also compared between centres. It would also inform the social and cost implications of treatment for patients and healthcare providers. Utilisation of prospective data collection tools like Medisoft and Fight Retinal Blindness! could play a pivotal role in facilitating the collection of this crucial real-world data. $\square$
1. Ting DS, Cheung GC, Wong TY, Diabetic retinopathy: global Tre major risk factors, screening practices and public health challenges: a revie, Clin Exp Ophthalmol, 2016:44:260-77.

2. Yau JW, Rogers SL, Kawasaki R, et al., Global prevalence and major risk factors of diabetic retinopathy, Diabetes Care, 2012;35:556-64.

3. Lee R, Wong TY, Sabanayagam C, Epidemiology of diabetic retinopathy, diabetic macular edema and related vision loss, Eye Vis (Lond), 2015;2:17.

4. Photocoagulation for diabetic macular edema. Early treatment diabetic retinopathy study report number 1 . Early treatment diabetic retinopathy study research group, Arch Ophthalmol 1985;103:1796-806

5. Virgili G, Parravano M, Menchini F, Evans JR, Anti-vascular endothelial growth factor for diabetic macular oedema, Cochrane Database Syst Rev, 2014:CD007419.

6. Grover D, Li TJ, Chong CC, Intravitreal steroids for macular edema in diabetes, Cochrane Database Syst Rev 2008:CD005656

7. Mehta H, Gillies M, Fraser-Bell S, Perspective on the role of Ozurdex (dexamethasone intravitreal implant) in the management of diabetic macular oedema, Ther Adv Chronic Dis, 2015;6:234-45

8. Avery RL, What is the evidence for systemic effects of intravitreal anti-VEGF agents, and should we be concerned? Br J Ophthalmol, 2014;98 Suppl 1:i7-10.

9. Bressler SB, Qin H, Beck RW, et al., Factors associated with changes in visual acuity and central subfield thickness at 1 yea after treatment for diabetic macular edema with ranibizumab, Arch Ophthalmol, 2012;130:1153-61.

10. Sivaprasad S, Oyetunde $S$, Impact of injection therapy on retinal patients with diabetic macular edema or retinal vein occlusion, Clin Ophthalmol, 2016;10:939-46.

11. Gillies MC, Sutter FK, Simpson JM, et al., Intravitreal triamcinolone for refractory diabetic macular edema: two-year results of a double-masked, placebo-controlled, randomized clinical trial, Ophthalmology, 2006;113:1533-8.

12. Gillies MC, Simpson JM, Gaston C, et al., Five-year results of a randomized trial with open-label extension of triamcinolone acetonide for refractory diabetic macular edema, Ophthalmology, 2009;116:2182-7.

13. Diabetic Retinopathy Clinical Research N, A randomized trial comparing intravitreal triamcinolone acetonide and focal/grid photocoagulation for diabetic macular edema, Ophthalmology, 2008;115:1447-9.

14. Diabetic Retinopathy Clinical Research N, Elman MJ, Aiello LP, et al., Randomized trial evaluating ranibizumab plus prompt or deferred laser or triamcinolone plus prompt laser for diabetic macular edema, Ophthalmology, 2010:117:1064-77.

15. Jonas JB, Degenring RF, Kamppeter BA, et al., Duration of the effect of intravitreal triamcinolone acetonide as treatment for diffuse diabetic macular edema, Am I Ophthalmol, 2004;138:158-60

16. Jonas JB, Spandau UH, Kamppeter BA, et al., Repeated intravitreal high-dosage injections of triamcinolone acetonide for diffuse diabetic macular edema, Ophthalmology, 2006;113:800-4

17. Smithen $\mathrm{LM}, \mathrm{Ober} \mathrm{MD}$, Maranan L, Spaide RF, Intravitreal triamcinolone acetonide and intraocular pressure, $\mathrm{Am} J$ Ophthalmol, 2004;138:740-3.

18. Chan $\mathrm{CK}$, Mohamed $\mathrm{S}$, Shanmugam MP, et al., Decreasing efficacy of repeated intravitreal triamcinolone injections in
diabetic macular oedema, Br $\mathrm{Ophthalmol,} \mathrm{2006;90:1137-41.}$

19. Larsson J, Zhu M, Sutter F, Gillies MC, Relation between reduction of foveal thickness and visual acuity in diabetic macular edema treated with intravitreal triamcinolone, $A m$ Ophthalmol, 2005:139:802-6.

20. Stepien KE, Eaton AM, Jaffe GJ, et al., Increased incidence of sterile endophthalmitis after intravitreal triamcinolone acetonide in spring 2006, Retina, 2009;29:207-13.

21. Pearson PA, Comstock TL, Ip M, et al., Fluocinolone acetonide intravitreal implant for diabetic macular edema: a 3-year multicenter, randomized, controlled clinical trial, Ophthalmology, 2011;118:1580-7.

22. Campochiaro PA, Brown DM, Pearson A, et al., Sustained delivery fluocinolone acetonide vitreous inserts provide benefit for at least 3 years in patients with diabetic macular edema, Ophthalmology, 2012;119:2125-32.

23. Alfagawi F, Lip PL, Elsherbiny S, et al., Report of 12-months efficacy and safety of intravitreal fluocinolone acetonide efficacy and safety of intravitreal fluocinolone acetonide
implant for the treatment of chronic diabetic macular oedema: a real-world result in the United Kingdom, Eye (Lond), oedema. a real-wo

24. Schmit-Eilenberger VK, A novel intravitreal fluocinolone acetonide implant (lluvien ${ }^{\circledast}$ ) in the treatment of patients with chronic diabetic macular edema that is insufficiently responsive to other medical treatment options: a case series, Clin Ophthalmol, 2015;9:801-11.

25. Elaraoud I, Andreatta W, Kidess A, et al., Use of flucinolone acetonide for patients with diabetic macular oedema: patient selection criteria and early outcomes in real world setting. BMC Ophthalmol, 2016;16:3.

26. Fluocinolone acetonide intravitreal implant for treating chronic diabetic macular oedema after an inadequate response to prio therapy. NICE Guidane. Available at: www.nice.org.uk/guidance/ ta301 (accessed 7 July 2017).
27. Figueira J, Henriques J, Amaro M, et al., A nonrandomized, open-label, multicenter, phase 4 pilot study on the effect and safety of ILUVIEN(R) in chronic diabetic macular edema patients considered insufficiently responsive to available therapies (RESPOND), Ophthalmic Res, 2017;57:166-72.

28. Chakravarthy U, Safety and clinical outcomes in the European registry study of ILUVIEN implant (fluocinolone acetonide; FAC) usage in diabetic macular edema. Presented at: EURetina congress Copenhagen, 8-11 September 2016.

29. Boyer DS, Yoon YH, Belfort R Jr., et al., Three-year, randomized, sham-controlled trial of dexamethasone intravitreal implant in patients with diabetic macular edema, Ophthalmology, 2014;121:1904-14

30. Callanan DG, Gupta S, Boyer DS, et al., Dexamethasone intravitreal implant in combination with laser photocoagulation for the treatment of diffuse diabetic macular edema Ophthalmology, 2013;120:1843-51.

31. Gillies MC, Lim LL, Campain A, et al., A randomized clinical trial of intravitreal bevacizumab versus intravitreal dexamethasone for diabetic macular edema: the BEVORDEX study, Ophthalmology, 2014;121:2473-81.

32. Fraser-Bell S, Lim LL, Campain A, et al., Bevacizumab or dexamethasone implants for DME: 2-year results (the BEVORDEX study), Ophthalmology, 2016;123:1399-401

33. Wickremasinghe SS, Fraser-Bell S, Alessandrello E, et al., Retinal vascular calibre changes after intravitreal bevacizumab or dexamethasone implant treatment for diabetic macular oedema, Br J Ophthalmol, 2017; doi: 10.1136/ bjophthalmol-2016-309882. [Epub ahead of print].

34. Mehta $\mathrm{H}$, Fraser-Bell S, Yeung A et al, Efficacy of dexamethasone versus bevacizumab on regression of hard exudates in diabetic maculopathy: data from the BEVORDEX

rand

5. Callanan DG, Loewenstein A, Patel SS, et al., A multicenter, 12-month randomized study comparing dexamethaso
intravitreal implant with ranibizumab in patients with intravitreal implant with ranibizumab in patients with
diabetic macular edema, Graefes Arch Clin Exp Ophthalmol, 2017;255:463-73.

36. Ramu J, Yang Y, Menon G, et al. A randomized clinical trial comparing fixed vs pro-re-nata dosing of Ozurdex in refractory diabetic macular oedema (OZDRY study), Eye (Lond), 2015;29:1603-12.

37. Ramu J, Chatziralli I, Yang Y, et al., Health-related quality of life, visual function and treatment satisfaction following intravitreal dexamethasone implant for diabetic macular edema, Patient Prefer Adherence, 2017;11:579-86.

38. Heng LZ, Sivaprasad S, Crosby-Nwaobi R, et al., A prospective randomised controlled clinical trial comparing a combination of 
repeated intravitreal Ozurdex and macular laser therapy versus macular laser only in centre-involving diabetic macular oedema (OZLASE study), Br $\lrcorner$ Ophthalmol, 2016:100:802-7.

39. Chhablani J, Bansal P, Veritti $D$, et al., Dexamethasone implan in diabetic macular edema in real-life situations, Eye (Lond), 2016;30:426-30

40. Aknin I, Melki L, Longitudinal study of sustained-release dexamethasone intravitreal implant in patients with diabetic macular edema, Ophthalmologica, 2016;235:187-8.

41. Malcles $A$, Dot $C$, Voirin $N$, et al., Real-life study in diabetic macular edema treated with dexamethasone implant: the Reldex study, Retina, 2017;37:753-60.

42. Malcles A, Dot C, Voirin N, et al., Safety of intravitreal dexamethasone implant (Ozurdex): the SAFODEX study. Incidence and Risk Factors of Ocular Hypertension, Retina, 2017;37:1352-9.

43. Matonti F, Pommier S, Meyer F, et al., Long-term efficacy and safety of intravitreal dexamethasone implant for the treatment of diabetic macular edema, Eur 1 Ophthalmol 2016;26:454-9.

44. Lam WC, Albiani DA, Yoganathan P, et al., Real-world assessment of intravitreal dexamethasone implant $(0.7 \mathrm{mg})$ in patients with macular edema: the CHROME study, Clin Ophthalmol, 2015;9:1255-68.

45. Escobar-Barranco JJ, Pina-Marin B, Fernandez-Bonet $M$, Dexamethasone implants in patients with naive or refractory diffuse diabetic macular edema, Ophthalmologica, 2015;233:176-85.

46. Guigou S, Haijar C, Parrat E, et al., Multicenter Ozurdex assessment for diabetic macular edema: MOZART study, $\mathrm{fF}$ .

47. Guigou S, Pommier S, Meyer F, et al., Efficacy and Safety of intravitreal Dexamethasone Implant in Patients with Diabetic Macular Edema, Ophthalmologica, 2015;233:169-75.

4. Bonnin S, Dupas B, Sanharawi ME, et al., Efficacy of dexamethasone intravitreal implant for the treatment of diabetic macular edema, Eur J Ophthalmol, 2015;25:448-53.

49. Dutra Medeiros M, Postorino M, Navarro R, et al., Dexamethasone intravitreal implant for treatment of patients with persistent diabetic macular edema, Ophthalmologica, 2014;231:141-6.

50. Bansal P, Gupta V, Gupta A, et al., Efficacy of Ozurdex implant in recalcitrant diabetic macular edema-a single-center experience, Int Ophthalmol, 2016;36:207-16.

51. Totan $Y$, Guler $E$, Guragac FB, Dexamethasone intravitrea implant for chronic diabetic macular edema resistant to intravitreal bevacizumab treatment, Curr Eye Res, 2016:41:107-13.

52. Khan Z, Kuriakose RK, Khan M, et al., Efficacy of the intravitreal sustained-release dexamethasone implant for diabetic macul edema refractory to anti-vascular endothelial growth factor therapy: meta-analysis and clinical implications, ophthalmic Surg Lasers Imaging Retina, 2017;48:160-6.

53. Khurana RN, Appa SN, McCannel CA, et al., Dexamethasone implant anterior chamber migration: risk factors, complications, and management strategies, Ophthalmology, 2014;121:67-71.

54. Keech AC, Mitchell P, Summanen PA, et al. .Effect of fenofibrate on the need for laser treatment for diabetic retinopathy (FIELD study): a randomised controlled trial, Lancet, 2007:370:1687-97. 5. Group AS, Group AES, Chew EY, et al., Effects of medica therapies on retinopathy progression in type 2 diabetes, N Eng Med, 2010:363:233-44

56. Nguyen QD, Brown DM, Marcus DM, et al., Ranibizumab for diabetic macular edema: results from 2 phase III randomized trials: RISE and RIDE, Ophthalmology, 2012;119:789-801.

57. Boyer DS, Faber D, Gupta S, et al., Dexamethasone intravitrea implant for treatment of diabetic macular edema in vitrectomized patients, Retina, 2011;31:915-23.

58. Yanyali A, Aytug B, Horozoglu F, Nohutcu AF, Bevacizumab (Avastin) for diabetic macular edema in previously vitrectomized eyes, Am J Ophthalmol, 2007;144:124-6.

59. Bressler SB, Melia M, Glassman AR, et al., Ranibizumab plus prompt or deferred laser for diabetic macular edema in eyes with vitrectomy before anti-vascular endothelial growth factor therapy, Retina, 2015;35:2516-28.

60. Heier IS Korobelnik JF, Brown DM et al, Intravitreal aflibercept for diabetic macular edema: 148-week results from the VISTA and VIVID studies, Ophthalmology, 2016;123:2376-85.

61. Holz FG, Bandello F, Gillies M, et al., Safety of ranibizumab in routine clinical practice: 1-year retrospective pooled analysis of four European neovascular AMD registries within the LUMINOUS programme, Br J Ophthalmol, 2013;97:1161-7.

62. Michaelides M, Kaines A, Hamilton RD, et al., A prospective randomized trial of intravitreal bevacizumab or laser therapy in the management of diabetic macular edema (BOLT study) 12-month data: report 2, Ophthalmology, 2010;117:1078-86. 